\title{
Characterization of salivary microbiome in patients with pancreatic cancer
}

Pedro J. Torres, Erin M. Fletcher, Sean M. Gibbons, Michael Bouvet, Kelly S. Doran, Scott T. Kelley

Clinical manifestations of pancreatic cancer often do not occur until the cancer has undergone metastasis resulting in a very low survival rate. In this study, we investigated whether salivary bacterial profiles might provide useful biomarkers for early detection of pancreatic cancer. Using high-throughput sequencing of bacterial small subunit ribosomal RNA (16S rRNA) gene, we characterized the salivary microbiota of patients with pancreatic cancer and compared them to healthy patients and patients with digestive or nondigestive diseases. A total of 146 patients were enrolled at the UCSD Moores Cancer Center where saliva and demographic data were collected from each patient. Of these, we analyzed the salivary microbiome of 108 patients: 8 had been diagnosed with pancreatic cancer, 13 with pancreatic disease, 53 with digestive diseases (including cancer), 12 with non-digestive diseases (including non-digestive cancer) and 22 were classified as nondiseased (healthy) controls. Bacterial 16S rRNA sequences were amplified directly from salivary DNA extractions and subjected to high-throughput sequencing (HTS). We found significant differences in the abundances of several bacterial genera between patients with pancreatic cancer and patients in other categories, including Porphyromonas, Haemophilus, and Campylobacter. In particular, we found the bacterial ratio of Leptotrichia to Porphyromonas was consistently higher in pancreatic cancer patients compared to those with pancreatic disease, other digestive diseases and healthy controls. Interestingly, we did not find lower abundances of Neisseria elongata and Streptococcus mitis or higher numbers of Granulicatella adiacens reported previously using microarray methods. While salivary bacterial biomarkers appear promising, larger sample studies and repeat sampling of individuals are needed to confirm the validity of particular markers. 
1 Characterization of the Salivary Microbiome in Patients with Pancreatic Cancer

2

3 Pedro J. Torres ${ }^{1}$, Erin M. Fletcher ${ }^{1}$, Sean M. Gibbons ${ }^{2,3}$, Michael Bouvet ${ }^{4}$, Kelly S. Doran ${ }^{1,5}$,

$4 \quad$ Scott T. Kelley ${ }^{1}$

5

$6{ }^{1}$ Department of Biology, San Diego State University, 5500 Campanile Drive, San Diego, CA 7 92182-4164, USA

$8{ }^{2}$ Graduate Program in Biophysical Sciences, University of Chicago, Chicago, IL 60637, USA

$9{ }^{3}$ Institute for Genomics and Systems Biology, Argonne National Laboratory, 9700, S. Cass Ave.

10 Lemont, IL 60439, USA

$11{ }^{4}$ Department of Surgery, University of California, 3855 Health Sciences Drive, La Jolla, CA 12 92093-0987, USA

$13{ }^{5}$ Department of Pediatrics, University of California San Diego School of Medicine, La Jolla, 14 CA, 92093, USA.

16 Running Title: Salivary Microbial Biomarkers for Pancreatic Cancer

18 \#Correspondence to: Dr. Scott T. Kelley, Department of Biology, San Diego State University,

195500 Campanile Drive, San Diego, CA 92182-4164, USA; Tel: 619-594-5371, Fax: 619-594-

20 5676, skelley@mail.sdsu.edu

21 Word count: 3,599

22

23 Total number of figures and tables: 7 


\section{Abstract}

31 Clinical manifestations of pancreatic cancer often do not occur until the cancer has undergone

32 metastasis, resulting in a very low survival rate. In this study, we investigated whether salivary

33 bacterial profiles might provide useful biomarkers for early detection of pancreatic cancer. Using

34 high-throughput sequencing of bacterial small subunit ribosomal RNA (16S rRNA) gene, we

35 characterized the salivary microbiota of patients with pancreatic cancer and compared them to

36 healthy patients and patients with other diseases, including pancreatic disease, non-pancreatic

37 digestive disease/cancer and non-digestive disease/cancer. A total of 146 patients were enrolled

38 at the UCSD Moores Cancer Center where saliva and demographic data were collected from

39 each patient. Of these, we analyzed the salivary microbiome of 108 patients: 8 had been

40 diagnosed with pancreatic cancer, 78 with other diseases and 22 were classified as non-diseased

41 (healthy) controls. Bacterial 16S rRNA sequences were amplified directly from salivary DNA

42 extractions and subjected to high-throughput sequencing (HTS). Several bacterial genera differed

43 in abundance in patients with pancreatic cancer. We found a significantly higher ratio of

44 Leptotrichia to Porphyromonas in the saliva of patients with pancreatic cancer than in the saliva

45 of healthy patients or those with other disease (Kruskal-Wallis Test; $\mathrm{P}<0.001)$. Leptotrichia

46 abundances were confirmed using real-time qPCR with Leptotrichia specific primers. Similar to 
47 previous studies, we found lower relative abundances of Neisseria and Aggregatibacter in the 48 saliva of pancreatic cancer patients, though these results were not significant at the $\mathrm{P}<0.05$ level 49 (K-W Test; $\mathrm{P}=0.07$ and $\mathrm{P}=0.09$ respectively). However, the relative abundances of other 50 previously identified bacterial biomarkers, e.g. Streptococcus mitis and Granulicatella adiacens, 51 were not significantly different in the saliva of pancreatic cancer patients. Overall, this study 52 supports the hypothesis that bacteria abundance profiles in saliva are useful biomarkers for 53 pancreatic cancer though much larger patient studies are needed to verify their predictive utility. 54 
55

56

57

58

59

60

61

62

63

64

65

\section{Introduction}

In the United States, approximately 40,000 people die every year from pancreatic adenocarcinoma, making it the fourth leading cause of cancer related death. Patients diagnosed in the early stage of pancreatic cancer have a 5-year survival rate of $24 \%$, compared to $1.8 \%$ when diagnosed in the advanced stage (1). Clinical manifestations of pancreatic cancer do not appear until after the cancer has undergone metastasis (2), emphasizing the need for early detection biomarkers. The etiology of pancreatic cancer remains elusive, with cigarette smoking being the most established risk factor (3-6), although links have also been made to diabetes (7; 8), obesity (9), and chronic pancreatitis (10). Recent research has also shown that men with periodontal disease have a two-fold greater risk of developing pancreatic cancer after adjusting for smoking, diabetes, and body mass index (11).

The human oral cavity harbors a complex microbial community (microbiome) known to contain over 700 species of bacteria, more than half of which have not been cultivated (12).

Researchers have identified a core microbial community in healthy individuals (13) and shifts from this core microbiome have been associated with dental carries and periodontitis (14). The composition of bacterial communities in saliva seems to reflect health status under certain circumstances (15), making the analysis of salivary microbiomes a promising approach for disease diagnostics. A study by Mittal et al. (2011) found that increases in the numbers of Streptococcus mutans and lactobacilli in saliva have been associated with oral disease prevalence (16), while another study showed that high salivary counts of Capnocytophaga gingivalis, Prevotella melaninogenica and Streptococcus mitis may be indicative of oral cancer (17).

A recent study by Farrell et al. (2012), suggested that the abundances of specific salivary bacteria could be used as biomarkers for early-stage pancreatic cancer. Using the Human Oral 
80 Microbe Identification Microarray (HOMIM), researchers observed decreased levels of Neisseria

81 elongata and Streptococcus mitis in patients with pancreatic cancer compared with healthy

82 individuals, while levels of Granulicatella adiacens were significantly higher in individuals with

83 pancreatic cancer (18). The HOMIM's ability to detect 300 of the most prevalent oral bacterial

84 species has made it a suitable method for assessing community profiles at the phylum level as

85 well as many common taxa at the genus level. However, the HOMIM microarray method fails to

86 detect approximately half of the bacterial species commonly present in saliva (19).

87 In this study, we applied high-throughput sequencing (HTS) of the bacterial small-

88 subunit ribosomal RNA (16S rRNA) genes to determine the salivary profiles of patients with and

89 without pancreatic cancer. The use of HTS to sequence 16S rRNA bacterial genes from entire

90 salivary microbial communities allows for a more comprehensive profile of the microbiome in

91 health and disease (20). During this study, we collected 146 saliva samples from patients at the

92 UCSD Moores Cancer Center. HTS was used to characterize the salivary microbiome of patients

93 with pancreatic cancer and compare them to patients with other diseases (including pancreatic

94 disease, non-pancreatic digestive disease/cancer and non-digestive disease/cancer) as well as

95 non-diseased (healthy) controls. This allowed us to test the hypothesis that patients with

96 pancreatic cancer may have a distinct microbial community profiles compared to non-diseased

97 controls and to other forms of digestive and non-digestive diseases. Our results demonstrated that

98 patients with pancreatic cancer had a significantly higher abundance ratio of particular bacterial

99 genera.

100

101 Materials and Methods

102 Sample collection and patient information 
104 Diego State University (SDSU) joint Institutional Review Board (IRB Approval \#120101).

105 Patients recruited for the study were being clinically evaluated at the UCSD Moores Cancer

106 Center or were undergoing endoscopy procedures by UCSD Gastroenterologists in the Thornton

107 Hospital Pre-Procedure Clinic between May 2012 and August 2013. All patients were required to

108 fast for 12-hours prior to cancer evaluation and endoscopy procedures. To avoid bias during

109 enrollment, the research coordinator responsible for recruiting participants' was unaware of

110 patient diagnosis at time of sample collection. Consenting participants were provided with IRB-

111 approved consent forms, and HIPAA forms, as well as an optional, voluntary written survey in

112 which they could share relevant information about antibiotic, dental and smoking history. All

113 participants gave informed consent and their identities were withheld from the research team.

114 Each subject was free to withdraw from the study at any time. Participants were asked to give a

115 saliva sample into a $50 \mathrm{~mL}$ conical tube. If the amount of saliva exceeded $55 \mathrm{uL}, 10 \mathrm{uL}$ was

116 transferred into tube containing Brain-Heart Infusion media (BHI) and glycerol for future

117 culturing. The remaining saliva was broken up into $55 \mathrm{uL}$ aliquots and stored in sterile cryovials.

118 Both BHI and saliva samples were then immediately stored at $-80^{\circ} \mathrm{C}$ until further processing.

119 Of the 146 participants, three subjects voluntarily withdrew and seven were not included

120 in the study due insufficient production of saliva $(<55 \mathrm{uL})$ leaving 136 saliva samples. After

121 sample collection, the research coordinator accessed the participants' medical records

122 electronically for patient diagnosis information that was included under a novel subject ID

123 number. Diagnosis was used to determine health status and assess the stage of disease when each

124 sample was taken. The various diagnoses were grouped into the following categories: pancreatic

125 cancer, other disease (including pancreatic disease, non-pancreatic digestive disease/cancer and 
126 non-digestive disease/cancer), and healthy (non-diseased) controls. Healthy individuals were

127 defined as participants with no documented chronic digestive or non-digestive disease, and a 5-

128 year resolution of any previously documented digestive or non-digestive disease. Exclusion

129 criteria included participants undergoing active chemotherapy or radiation therapy or use of

130 antibiotics two weeks prior to saliva collection as well as invasive surgery in the past year.

\section{DNA Isolation, PCR and 16S rRNA Sequencing}

Bacterial DNA was extracted directly from $50 \mathrm{uL}$ of patient saliva using the MoBio

PowerSoil DNA Extraction Kit (Catalogue 12888-05, Mo Bio Laboratories, Carlsbad, CA, USA)

following the manufacturer's protocol. Genomic DNA was quantified using the NanoDrop ${ }^{\mathrm{TM}}$

136 Spectrophotometer and stored at $-20^{\circ} \mathrm{C}$.

137 The 16S ribosomal RNA (rRNA) amplicon region was amplified using barcoded 'universal'

138 bacterial primer 515F (5'- AATGATACGGCGACCACCGAGATCTACAC TATGGTAATT

139 GT GTGCCAGCMGCCGCGGTAA-3') and 806R (5'-

140 CAAGCAGAAGACGGCATACGAGAT XXXXXXXXXXXX AGTCAGTCAG CC

141 GGACTACHVGGGTWTCTAAT-3') (X's indicate the location of the 12-bp barcode) with

142 Illumina adaptors used by the Earth Microbiome Project (http://www.earthmicrobiome.org/emp-

143 standard-protocols/16s). The barcoded primers allow pooling of multiple PCR amplicons in a

144 single sequencing run. PCR was carried out using the reaction conditions outlined by the Earth

145 Microbiome Project. Thermocycling parameters were as follows: $94^{\circ} \mathrm{C}$ for 3 minutes

146 (denaturing) followed by amplification for 35 cycles at $94^{\circ} \mathrm{C}$ for $45 \mathrm{~s}, 50^{\circ} \mathrm{C}$ for $60 \mathrm{~s}$ and $72^{\circ} \mathrm{C}$ for

$14790 \mathrm{~s}$, and a final extension of $72^{\circ} \mathrm{C}$ for 10 minutes (21). PCR amplicons were then sequenced on 
148 the Illumina MiSeq platform at the Argonne National Laboratory Core sequencing facility

149 (Lemont, IL).

150

151 Sequence Analysis

152 16S rRNA sequences were de-multiplexed using the Quantitative Insights Into Microbial

153 Ecology (QIIME v. 1.8.0, http://www.qiime.org) pipeline. Sequences were grouped into

154 operational taxonomic units (OTUs) at 97\% sequence similarity using the Greengenes reference

155 database. OTUs that did not cluster with known taxa at $97 \%$ identity or higher in the database

156 were clustered de novo (UCLUST (22)). Representative sequences for each OTU were then

157 aligned using PyNast (23), and taxonomy was assigned using the RDP classifier (Version 2.2)

158 (24). A phylogenetic tree was built using FastTree (25). Before performing downstream analysis,

159 patient samples were rarefied to 10,0000 sequences per sample, singletons and OTUs present in

$160<25 \%$ of samples were removed prior to rarefaction. Chimeric sequences were identified using

161 ChimeraSlayer in QIIME, as well as with DECIPHER (26), and subsequently removed. Alpha

162 diversity metrics were computed using QIIME. Beta diversity distance between samples

163 (weighted and unweighted UniFrac) were computed and used to account for both differences in

164 relative abundance of taxa and phylogeny (27). Beta diversity comparisons were done using

165 analysis of similarities (ANOSIM). We also tested whether there were significant differences in

166 abundance ratios of particular genera between our different categories with GraphPad Prism

167 version 6.0 using the Kruskal-Wallis test followed by Dunn's multiple comparison correction.

168 Statistical significance was accepted at a $p<0.05$. Analysis and identification of potential

169 contaminants was done using SourceTracker (28).

170 Quantitative PCR (qPCR) 
172 estimated Leptotrichia abundance using Leptotrichia specific 16S primers and normalized their

173 values to overall bacterial abundance estimated using qPCR with universal bacterial $16 \mathrm{~S}$ primers

174 (5'-TCCTACGGGAGGCAGCAGT-3' forward primer, and 5'-

175 GGACTACCAGGGTATCTAATCCTGTT-3' reverse primer) developed by Nadkarni et al.

176 (2002) (29). qPCR was performed on a Bio-Rad CFX96 Touch ${ }^{\mathrm{TM}}$ Real-Time PCR Detection

177 Instrument. The maximum $\mathrm{C}_{\mathrm{t}}$ (threshold cycle) for the universal $16 \mathrm{~S}$ primers was set to 35 cycles

178 and $\mathrm{C}_{\mathrm{t}}$ levels above this threshold were considered background noise. Genus-specific primers for

179 amplification of Leptotrichia were designed using 16S rRNA sequences obtained from the RDP

180 classifier (Version 2.2) (24). Primer3 online software was used for primer selection, and

181 conditions were settled following the recommendations of Thorton and Basu, 2011 (30). The

182 Leptotrichia forward primer sequence (5'- GGAGCAAACAGGATTAGATACCC - 3') and the

183 Leptotrichia reverse primer sequence (5'- TTCGGCACAGACACTCTTCAT- 3') generated an

184 amplicon of $87 \mathrm{bp}$. The PCR reaction contained $1 \mathrm{uM}$ of both forward and reverse Leptotrichia

185 primers with thermocycling parameters of $50^{\circ} \mathrm{C}$ for $2 \mathrm{~min}, 95^{\circ} \mathrm{C}$ for $10 \mathrm{~min}$ and 40 cycles of

$18695^{\circ} \mathrm{C}$ for $15 \mathrm{sec}$ and $62.5^{\circ} \mathrm{C}$ for $1 \mathrm{~min}$. The amplification reactions for the universal primers and

187 Leptotrichia primers were carried out in at least duplicate using $25 \mathrm{uL}$ of SYBR Green Master

188 Mix (Bio-Rad) and $0.85 \mathrm{ng} / \mathrm{uL}$ of extracted DNA as template. Various online tools, including In

189 silico PCR Amplification (31) and Ribosomal Database Project (24) were used to check the

190 specificities of the oligonucleotide primer sequences for the target organism. A saliva sample

191 was sequenced (Eton Bioscience, San Diego, CA) using our novel primers and primer specificity

192 was further confirmed with a 16S rRNA database BlastN search. 
194 Results

195 Salivary microbial diversity profiles were generated for a total of 108 patients. 8 patients

196 were diagnosed with pancreatic cancer (P), 78 were diagnosed with other diseases (including

197 cancer) (O), and 22 were considered healthy (non-diseased) controls (H). Table 1 details the

198 individual clinical characteristics, including, gender and ethnicity. Of the 108 patients, 23

199 patients in pancreatic, digestive, and non-digestive disease categories were diagnosed with

200 having cancer. Table 2 details the types of cancer, as well as category groupings and the mean

201 age of the cancer patients in each category.

202 Illumina sequencing yielded approximately 6.8 million sequences across all samples. The

203 sequences are available on FigShare (http://dx.doi.org/10.6084/m9.figshare.1422174) along with

204 the mapping file (http://dx.doi.org/10.6084/m9.figshare.1422175). An analysis of potential

205 sample contamination using SourceTracker (28) identified some evidence of human skin and/or

206 environmental contamination. The sequences associated with OTUs identified as contaminants,

207 mostly Staphylococcus (skin) and Cyanobacteria (chloroplasts), were removed from all

208 subsequent analyses. From these data, we identified a total of 12 bacterial phyla and 139 genera.

209 Proteobacteria, Actinobacteria, Bacteroidetes, Firmicutes, and Fusobacteria were the 5 major

210 phyla, accounting for $99.3 \%$ of oral bacteria (Figure 1). The mean relative abundance of

211 Proteobacteria was lower in pancreatic cancer patients relative to other sample categories, while

212 Firmicutes tended to be higher, though these were not significant after adjusting for multiple

213 comparisons (FDR). The pancreatic cancer group also had higher levels of Leptotrichia, as well

214 as lower levels of Porphyromonas, and Neisseria (Figure 2). In general, multi-level taxonomic

215 profiles of the healthy group resembled the 'other' disease group, while the pancreatic cancer

216 group was readily distinguishable (Fig. S1). However, there were no significant differences 
217 among the three main groupings $(\mathrm{H}, \mathrm{O}$, and $\mathrm{P}$ ) in either beta diversity (ANOSIM; $\mathrm{P}=0.1$ ) or

218 alpha diversity (Chao1, K-W test; $\mathrm{P}=0.6$; Faith's $\mathrm{PD}, \mathrm{K}-\mathrm{W}$ test; $\mathrm{P}=0.56$ ).

219 As in previous studies by Farrell et al. (2012) and Lin et al. (2013) (32), we saw lower

220 relative abundances of Neisseria and Aggregatibacter, although these differences were not

221 significant ( $\mathrm{K}-\mathrm{W}$ test; $\mathrm{P}=0.07$ and $\mathrm{P}=0.09$ respectively). Bacteriodes was more abundant in

222 pancreatic cancer patients compared to healthy individuals, similar to what Lin et al. observed,

223 although this too was not significant $(\mathrm{K}-\mathrm{W}$ test; $\mathrm{P}=0.27)$. We did not see any difference in the

224 relative abundance of Streptococcus or Granulicatella, which were shown to differ in a prior

225 pancreatic cancer study (18). Additional analytical targets were based on a preliminary study

226 consisting of our first 61 saliva samples (including 3 from pancreatic cancer patients), which

227 showed significantly higher Leptotrichia and lower Porphyromonas in pancreatic cancer patient 228 saliva.

The abundance ratio of Leptotrichia, specifically two OTUs (arbitrarily named OTU 23031235 and OTU 4443207), to Porphyromonas was significantly higher in pancreatic cancer

231 patients (Figure 3). A BLAST comparison of these OTUs to the 16S sequence in the Human Oral

232 Microbiome database (33) (HOMD RefSeq Version 13.2) found OTU 31235 to be $100 \%$ similar

233 to Leptotrichia sp. Oral taxon 221, while OTU 4443207 was $99.3 \%$ similar to Leptotrichia

234 hongkongensis. We found a strong positive correlation (Pearson's correlation $\mathrm{r}=0.903$,

$235 \mathrm{P}=0.0000001)$ between Leptotrichia abundances obtained from $16 \mathrm{~S}$ rRNA sequencing (OTU

236 relative abundances) and from real-time qPCR (Figure 4). 


\section{Discussion}

239 Our analysis of salivary microbial profiles supports prior work suggesting that salivary

240 microbial communities of patients diagnosed with pancreatic cancer are distinguishable from

241 salivary microbial communities of healthy patients or patients with other diseases, including non-

242 pancreatic cancers. At the phylum level, pancreatic cancer patients tended to have higher

243 proportions of Firmicutes and lower proportions of Proteobacteria (Figure 1). At finer taxonomic

244 levels, we observed differences in the mean relative abundances of particular genera in

245 pancreatic cancer patients compared to other patient groups (Figure 2). For instance, there was a

246 higher proportion of Leptotrichia in pancreatic cancer patients, while the proportion of

247 Porphyromonas and Neisseria were lower in these patients.

248 The most striking difference we found between the microbial profiles of pancreatic

249 cancer patients and other patient groups was in the ratio of the bacterial genera Leptotrichia and

250 Porphyromonas (LP ratio) (Figure 3). The LP ratio had been identified as a potential biomarker

251 from a preliminary analysis and an analysis of the full dataset found significantly higher LP ratio

252 in pancreatic cancer patient saliva than in other patient groups. To verify these differences using

253 another method, we cross-validated the relative abundances of Leptotrichia (Figure 4).

254 Interestingly, during the analysis of the 16S rRNA data, we successfully used the LP ratio to

255 reclassify one of the patients in the non-pancreatic cancer disease group. This particular

256 individual had been initially diagnosed as having an unknown digestive disease, but the patient's

257 high LP ratio suggested pancreatic cancer (Figure 3). Subsequently, the patient was re-evaluated 258 and diagnosed with pancreatic cancer, supporting the notion that the LP ratio may serve as a 259 pancreatic cancer biomarker. 
Despite the small cohort of patients in this study, we believe our results are especially

261 noteworthy because we were able to distinguish between patients with pancreatic cancer and

262 patients with a variety of other diseases (including non-pancreatic cancer), in addition to healthy

263 controls. Other researchers have proposed the use of ratios of bacterial taxa previously.

264 Galimanas et al. (2014) suggested using salivary bacteria abundance ratios as a means for

265 differentiating between healthy and diseased patients (34). Taxonomic ratios have been used to

266 differentiate between subjects in studies of obesity (35), diabetes (36), and periodontal disease

267 (37). Ratio comparisons also help to control for high levels of taxonomic variability among 268 individuals (38-41).

A review of the literature revealed that Leptotrichia's role in oral health remains elusive. However, these bacteria have been found in the bloodstream of immune-compromised patients (42) and co-occur significantly with colorectal tumors (43). Leptotrichia have been isolated from 272 cardiovascular and gastrointestinal abscesses, from systemic infections, and are thought to be 273 pathogenic (44). In regards to Porphyromonas, antibodies to Porphyromonas gingivalis have been directly associated with pancreatic cancer (45). A European cohort study measured plasma antibodies to 25 oral bacteria in pre-diagnostic blood samples from 405 pancreatic cancer patients and 416 matched controls and found a $>2$-fold increase in risk of pancreatic cancer among those with higher antibody titers to a pathogenic strain of $P$. gingivalis (45). At first 278 glance, it appears contradictory that individuals with higher Porphyromonas antibody titers would have lower oral abundances. However, studies of systemic immunization of animals to 280 particular periopathogens including Porphyromonas have shown reduced colonization of these 
282 antibody titers in individuals with pancreatic cancer may decrease their oral abundance, though

283 this connection needs to be formally tested.

284 Shifts in salivary microbial diversity could also be a systematic response to pancreatic

285 cancer. Pancreatic cancer is known to weaken the immune system (49), which could lead to

286 overgrowth of oral bacteria and a shift towards systemically invasive periodontal pathogens. The

287 proliferation of bacterial pathogens could assist cancer progression through systemic

288 inflammation (50) or immune distraction (51). Thus, an initial increase in Porphyromonas might

289 be followed by a decrease due to systemic invasion and antibody production. Indeed,

290 inflammation is thought to play a significant role in the development of pancreatic cancer (52).

291 We also compared the relative abundances of several other bacterial genera that were

292 indicated as potential biomarkers in previous work by Farrell et al. (2012). Like Farrell et al., we

293 found a lower proportion of Neisseria in pancreatic cancer patient saliva compared with the

294 healthy and other disease category, though this trend was not significant. However, we did not

295 find the same results as Farrell et al. for the other bacterial genera they identified. Our data also

296 showed an increase in Bacteroides and decrease in the abundance of the bacterial genus

297 Aggregatibacter in patients with pancreatic cancer, supporting the results of a pilot study by Lin

298 et al. (2013), though neither trends were significant.

Methodological differences between our study and the Farrell et al. study in particular,

300 may partially explain our divergent results. For instance, the inability of the V4 region of the16S

301 rRNA gene to discriminate Streptococcus mitis from other Streptococcus species may have

302 prevented us from detecting difference in this species' abundance (18). Additionally, our study

303 had a broader array of patient categories and cancers were not always confined to the pancreas at

304 the time of sampling. 

detection of oral Fusobacterium in pancreatic cancer tissue (53). A retrospective review of our abundance data also found a lower relative abundance of Fusobacterium in pancreatic cancer patients compared to other patient categories (Figure $2 ; \mathrm{K}-\mathrm{W}$ test, $\mathrm{P}=0.03$ prior to $\mathrm{FDR}$ correction) suggesting the processes driving differences in Fusobacterium may be similar to our proposed mechanism for Porphyromonas. Although the result was not significant after adjusting

311 for multiple-comparisons (FDR), we suggest Fusobacterium abundance should be considered as

312 a potential biomarker target for future studies with larger patient cohorts.

313 Overall, our study suggests that members of the salivary microbiome have promise as

314 potential pancreatic cancer biomarkers and we may have uncovered an important new prospect

315 in this regard (i.e., the LP ratio). However, our relatively small number of samples from

316 pancreatic cancer patients and the discrepancies between our findings and previous work indicate

317 that much larger patient cohorts will be needed to determine whether salivary biomarkers are

318 diagnostically useful. Future studies should focus on improved metadata collection, including

319 diet and oral health information (i.e. periodontal disease), which would make it possible to run

320 statistical analyses that control for multiple factors involved in shaping oral microbial diversity.

321 It will also be important to sample the same individual's saliva over time to assess whether we

322 can distinguish between disease stages and also to control for intra-individual variation. Further,

323 it is possible that single biomarkers may never be able to consistently identify pancreatic patients

324 from other conditions. Thus, we may need more complex metrics that combine the abundances

325 of multiple salivary bacteria, metabolite profiles, and detailed patient metadata. Effective

326 diagnostic biomarkers for pancreatic cancers have been difficult to find, but are sorely needed

327 and have the potential to save thousands of lives each year. 


\section{Acknowledgements}

330 We thank S. Owens and J. Marcel for their help in our sequencing runs. We also thank M.

331 Wachter for assistance in enrolling patients into the study. Thanks to all the patients who

332 volunteered to be a part of this study.

\section{References:}

336 1. Li D, Xie K, Wolff R, Abbruzzese JL. Pancreatic cancer. Lancet 2004;363:1049-57.

337 2. Holly EA, Chaliha I, Bracci PM, Gautam M. Signs and symptoms of pancreatic 338 cancer: a population-based case-control study in the San Francisco Bay area. Clin Gastroenterol $339 \quad H$ 2004;2:510-17.

340 3. Vrieling A, Bueno-de-Mesquita HB, Boshuizen HC, Michaud DS, Severinsen

341 MT, Overvad K, Olsen A, Tjønneland A,Clavel-Chapelon F, Boutron-Ruault MC, Kaaks

342 R, Rohrmann S, Boeing H, Nöthlings U, Trichopoulou A, Moutsiou E, Dilis V, Palli D, Krogh

343 V, Panico S, Tumino R, Vineis P, van Gils CH, Peeters PH, Lund E, Gram IT, Rodríguez

344 L, Agudo A,Larrañaga N, Sánchez MJ, Navarro C, Barricarte A, Manjer J, Lindkvist B, Sund

345 M, Ye W, Bingham S, Khaw KT, Roddam A, Key T, Boffetta P, Duell EJ, Jenab M, Gallo

346 V, Riboli E.Cigarette smoking, environmental tobacco smoke exposure and pancreatic cancer

347 risk in the European Prospective Investigation into Cancer and Nutrition. Int J Cancer

$348 \quad 2010 ; 126: 2394-2403$.

349 4. Nakamura K, Nagata C, Wada K, Tamai Y, Tsuji M, Takatsuka N, Shimizu H. Cigarette

350 Smoking and Other Lifestyle Factors in Relation to the Risk of Pancreatic Cancer Death: A 
351 Prospective Cohort Study in Japan. Jpn J Clin Oncol 2011;41:225-231.

352 5. Fuchs CS, Colditz GA, Stampfer MJ. A prospective study of cigarette smoking and the 353 risk of pancreatic cancer. Arch Intern Med 1996;156:2255-60.

354 6. Zheng W, McLaughlin JK, Gridley G, Bjelke E. A cohort study of smoking, alcohol 355 consumption, and dietary factors for pancreatic cancer (United States). Cancer Cause Control $356 \quad 1993 ; 4: 477-82$.

357 7. Haugvik S-PP, Hedenström P, Korsæth E, Valente R, Hayes A, Siuka D, Maisonneuve 358 P, Gladhaug IP, Lindkvist B,Capurso G.Diabetes, Smoking, Alcohol and Family History of

359 Cancer as Risk Factors for Pancreatic Neuroendocrine Tumors: A Systematic Review and Meta360 Analysis. Neuroendocrinology 2015 Jan 22. [Epub ahead of print].

361 8. Liu X, Hemminki K, Försti A, Sundquist K, Sundquist J, Ji J. Cancer risk in patients with 362 type 2 diabetes mellitus and their relatives. Int J Cancer 2015 Jan 21. [Epub ahead of print].

363 9. Bracci PM. Obesity and pancreatic cancer: overview of epidemiologic evidence and 364 biologic mechanisms. Mol Carcinogen 2012;51:53-63.

365 10. Malka D, Hammel P, Maire F, Rufat P, Madeira I. Risk of pancreatic adenocarcinoma 366 in chronic pancreatitis. Gut 2002;51:849-52.

367 11. Michaud DS, Joshipura K, Giovannucci E, Fuchs CS. A prospective study of periodontal 368 disease and pancreatic cancer in US male health professionals. J Natl Cancer I 2007;99:171-5.

369 12. Aas JA, Paster BJ, Stokes LN, Olsen I. Defining the normal bacterial flora of the oral 370 cavity. J Clin Microbiol 2005;43:5721-32.

371 13. Zaura E, Keijser B, Huse SM. Defining the healthy "core microbiome" of oral microbial 372 communitites. BMC Microbiol 2009;9:259.

373 14. Berezow AB, Darveau RP. Microbial shift and periodontitis. Periodontol 2000 
$374 \quad 2011 ; \mathbf{5 5}: 36-47$.

375 15. Yamanaka W, Takeshita T, Shibata Y, Matsuo K. Compositional stability of a salivary

376 bacterial population against supragingival microbiota shift following periodontal therapy. PLoS

377 One 2012;7:e42806.

378 16. Mittal S, Bansal V, Garg S, Atreja G, Bansal S. The diagnostic role of Saliva-A Review.

379 J Clin Exp Dent 2011;3:e314-20.

380 17. Mager D, Haffajee A, Devlin P, Norris C, Posner M, Goodson J. The salivary microbiota

381 as a diagnostic indicator of oral cancer: A descriptive, non-randomized study of cancer-free and

382 oral squamous cell carcinoma subjects. $J$ Transl Med 2005;3:27.

383 18. Farrell J, Zhang L, Zhou H, Chia D, Elashoff D, Akin D, Paster BJ, Joshipura K, Wong

384 DT. Variations of oral microbiota are associated with pancreatic diseases including pancreatic

385 cancer. Gut 2012;61:582-8.

386 19. Ahn J, Yang L, Paster BJ, Ganly I, Morris L, Pei Z. Oral microbiome profiles: 16S

387 rRNA pyrosequencing and microarray assay comparison. PLoS One 2011;6:e22788.

388 20. Kuczynski J, Lauber CL, Walters WA. Experimental and analytical tools for studying

389 the human microbiome. Nat Rev Genet 2011;13:47-58.

390 21. Caporaso J, Lauber C, Walters W, Lyons-Berg D, Lozupone CA, Turnbaugh PJ, Fierer

391 N, Knight R. Global patterns of 16S rRNA diversity at a depth of millions of sequences per

392 sample. P Natl Acad Sci USA 2011;108:4516-22.

393 22. Edgar RC. Search and clustering orders of magnitude faster than BLAST. Bioinformatics

394 2010;26:2460-1.

395 23. Caporaso JG, Bittinger K, Bushman FD. PyNAST: a flexible tool for aligning

396 sequences to a template alignment. Bioinformatics 2010;26:266-7 
397 24. Cole JR, Chai B, Marsh TL, Farris RJ. The Ribosomal Database Project (RDP-II):

398 previewing a new autoaligner that allows regular updates and the new prokaryotic taxonomy.

399 Nucleic Acids Res 2003;31:442-3

400 25. Price MN, Dehal PS, Arkin AP. FastTree: computing large minimum evolution trees with 401 profiles instead of a distance matrix. Mol Biol Evol 2009;26:1641-50.

402 26. Wright ES, Yilmaz LS, Noguera DR. DECIPHER, A Search-Based Approach to Chimera 403 Identification for 16S rRNA Sequences. Appl Environ Microbiol 2012;

404 doi:10.1128/AEM.06516-11.

405 27. Vázquez-Baeza Y, Pirrung M, Gonzalez A, Knight R. EMPeror: a tool for visualizing 406 high-throughput microbial community data. Gigascience 2013;2:16.

407 28. Knights D, Kuczynski J, Charlson ES, Zaneveld J, Mozer MC, Collman RG, Bushman 408 FD, Knight R, Kelley ST. Bayesian community-wide culture-independent microbial source 409 tracking. Nat Methods 2011;8:761-3.

410 29. Nadkarni MA, Martin FE, Jacques NA, Hunter N. Determination of bacterial load by

411 real-time PCR using a broad-range (universal) probe and primers set. Microbiol-UK

$412 \quad 2002 ; 148: 257-66$.

413 30. Thornton B, Basu C. Real-time PCR (qPCR) primer design using free online software.

414 Biochem Mol Biol Edu 2011;39:145-54.

415 31. Bikandi J, San Millán R, Rementeria A, Garaizar J. In silico analysis of complete

416 bacterial genomes: PCR, AFLP-PCR and endonuclease restriction. Bioinformatics 2004;20:7984179.

418 32. Lin IH, Wu J, Cohen, SM, Chen C, Bryk D, Marr M, Melis M, Newman E, Patcher HL, 419 Alekseyenko AV, Hayes RB, Ahn J. Abstracts 101: Pilot study of oral microbiome and risk of 
420 pancreatic cancer. Cancer Res 2013; doi: 10.1158/1538-7445.AM2013-101.

421 33. Chen T, Yu W-HH, Izard J, Baranova OV, Lakshmanan A, Dewhirst FE. The Human

422 Oral Microbiome Database: a web accessible resource for investigating oral microbe taxonomic 423 and genomic information. Database 2010;2010:baq013.

424 34. Galimanas V, Hall MW, Singh N, Lynch MD, Goldberg M, Tenenbaum H, Cvitkovitch 425 DG, Neufeld JD,Senadheera DB. Bacterial community composition of chronic periodontitis and 426 novel oral sampling sites for detecting disease indicators. Microbiome 2014;2:32.

427 35. Lazarevic V, Whiteson K, Gaïa N, Hernandez D, Farinelli L, Osterås M, François

428 P, Schrenzel J.Analysis of the salivary microbiome using culture-independent techniques. $J$ Clin 429 Bioinforma 2012;2:4.

430 36. Zhang Y, Zhang H. Microbiota associated with type 2 diabetes and its related 431 complications. Food Sci Hum Wellness 2013;2:167-72.

432 37. Moolya NN, Shetty A, Gupta N, Gupta A, Jalan V, Sharma R. Orthodontic bracket 433 designs and their impact on microbial profile and periodontal disease: A clinical trial. $J$ Orthod 434 Sci 2014;3:125-31.

435 38. Ding T, Schloss PD. Dynamics and associations of microbial community types across 436 the human body. Nature 2014;509:357-60.

437 39. Grice EA, Segre JA. The human microbiome: our second genome. Annu Rev Genom 438 Hum G 2012;13:151-70.

439 40. Schwarzberg K, Le R, Bharti B, Lindsay S, Casaburi G, Salvatore F, Saber MH,

440 Alonaizan F, Slots J, Gottlieb RA, Caporaso JG, Kelley ST. The Personal Human Oral

441 Microbiome Obscures the Effects of Treatment on Periodontal Disease. PLoS One $442 \quad$ 2014;9:e86708. 
443 41. Wang J, Qi J, Zhao H, He S, Zhang Y, Wei S, Zhao F. Metagenomic sequencing

444 reveals microbiota and its functional potential associated with periodontal disease. Sci Rep $445 \quad 2013 ; 3: 1843$.

446 42. Eribe E, Olsen I. Leptotrichia species in human infections. Anaerobe 2008;14:131-7.

447 43. Warren RL, Freeman DJ, Pleasance S, Watson P, Moore RA, Cochrane K, Allen-Vercoe

448 E, Holt RA. Co-occurrence of anaerobic bacteria in colorectal carcinomas. Microbiome $449 \quad 2013 ; \mathbf{1}: 16$.

450 44. Han YW, Wang X. Mobile Microbiome Oral Bacteria in Extra-oral Infections and 451 Inflammation. J Dent Res 2013;92:485-91.

452 45. Michaud DS, Izard J, Wilhelm-Benartzi CS, You DH, Grote VA, Tjønneland A, Dahm 453 CC, Overvad K, Jenab M, Fedirko V,Boutron-Ruault MC, Clavel-Chapelon F, Racine A, Kaaks 454 R, Boeing H, Foerster J, Trichopoulou A, Lagiou P, Trichopoulos D, Sacerdote C, Sieri S, Palli 455 D, Tumino R, Panico S, Siersema PD, Peeters PH, Lund E, Barricarte A, Huerta JM, Molina456 Montes E, Dorronsoro M, Quirós JR, Duell EJ, Ye W, Sund M, Lindkvist B, Johansen D, Khaw 457 KT, Wareham N, Travis RC,Vineis P, Bueno-de-Mesquita HB, Riboli E. Plasma antibodies to 458 oral bacteria and risk of pancreatic cancer in a large European prospective cohort study. Gut $459 \quad 2012 ; 62: 1764-70$.

460 46. Evans RT, Klausen B, Sojar HT, et al. Immunization with Porphyromonas (Bacteroides) 461 gingivalis fimbriae protects against periodontal destruction. Infect Immun 1992;60:2926-35.

462 47. Persson GR, Engel D, Whitney C, Darveau R, Weinberg A, Brunsvold M, Page RC.

463 Immunization against Porphyromonas gingivalis inhibits progression of experimental

464 periodontitis in nonhuman primates. Infect Immun 1994;62:1026-31.

465 48. Clark WB, Magnusson I, Beem JE, Jung JM, Marks RG, McArthur WP. Immune 
466 modulation of Prevotella intermedia colonization in squirrel monkeys. Infect Immun

467 1991;59:1927-31.

468 49. von Bernstorff W, Voss M, Freichel S, Schmid A. Systemic and local

469 immunosuppression in pancreatic cancer patients. Clin Cancer Res 2001;7:925-32s.

470 50. El-Shinnawi U, Soory M. Associations between periodontitis and systemic

471 inflammatory diseases: response to treatment. Recent Pat Emdocr Metab Immune Drug Discov

$472 \quad 2013 ; 7: 169-88$.

473 51. Feurino LW, Zhang Y, Bharadwaj U. IL-6 stimulates Th2 type cytokine secretion and

474 upregulates VEGF and NRP-1 expression in pancreatic cancer cells. Cancer Biol Ther

$475 \quad 2007 ; 6: 1096-100$.

476 52. Farrow B, Evers MB. Inflammation and the development of pancreatic cancer. Surg

477 Oncol 2002;10:153-69.

478 53. Mitsuhashi K, Nosho K, Sukawa Y, Matsunaga Y, Ito M, Kurihara H, Kanno S, Igarashi

479 H, Naito T, Adachi Y,Tachibana M, Tanuma T, Maguchi H, Shinohara T, Hasegawa T, Imamura

480 M, Kimura Y, Hirata K, Maruyama R, Suzuki H, Imai K, Yamamoto H, Shinomura Y.

481 Association of Fusobacterium species in pancreatic cancer tissues with molecular features and

482 prognosis. Oncotarget 2015;30:7209-20. 


\section{Figure 1 (on next page)}

Relative abundance of phyla identified in patient saliva summarized by diagnosis group.

Relative abundance of phyla in oral communities from 108 study patients summarized by diagnosis group $(\mathrm{H}=$ healthy control, $\mathrm{O}=$ other disease, and $\mathrm{P}=$ pancreatic cancer). 


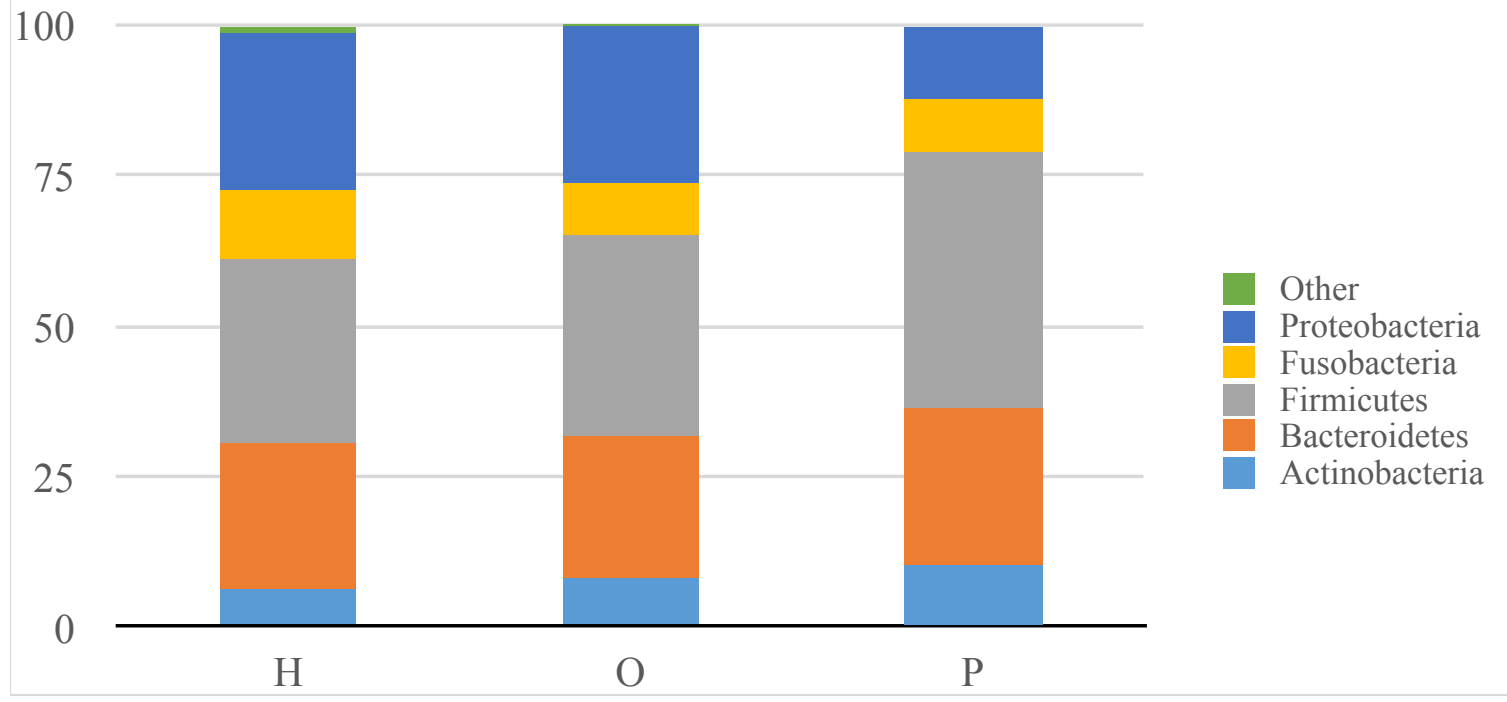




\section{Figure 2 (on next page)}

Mean relative abundances of particular genera in pancreatic cancer patients $(\mathrm{P})$ compared to healthy $(\mathrm{H})$ and other disease $(\mathrm{O})$ patient groups.

Relative abundances of genera in oral communities from 108 patients. Arrows point to specific genera that showed interesting trends across diagnosis groups.[b] 

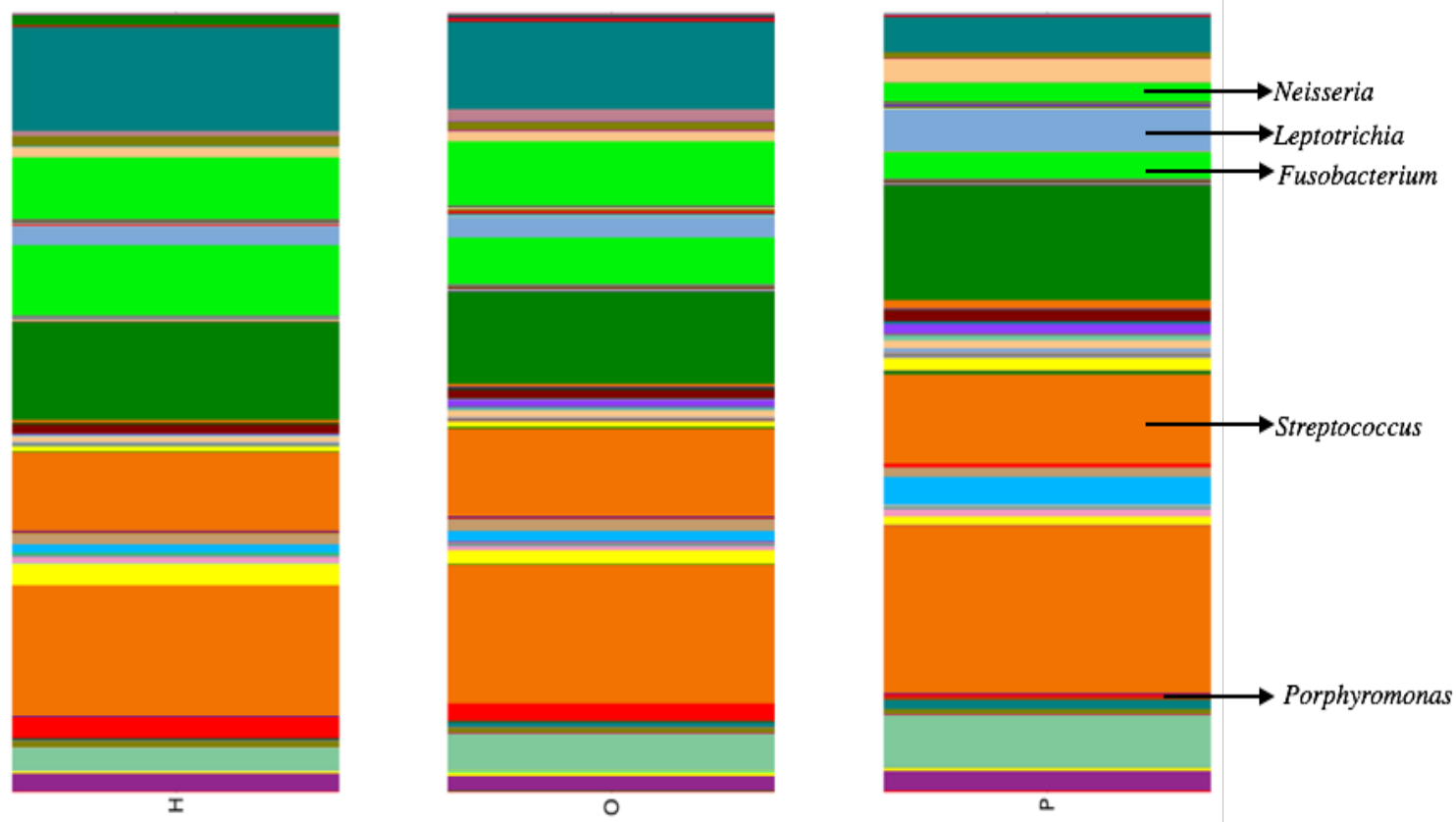
Figure 3 (on next page)

Abundance ratio of Leptotrichia to Porphyromonas between different patient categories.

Each symbol represents the ratio of Leptotrichia Oral Taxon 221 and Leptotrichia hongkongenesis to Porphyromonas for an individual patient $(n=108)$. Patients are grouped into 3 different categories depending on their diagnosis: healthy control $(H)$, other diseases (including cancer) ( 0 ), and pancreatic cancer $(P)$. Horizontal bar and error bars represent the mean and SEM, respectively. ${ }^{* * *} p<0.001$ (Kruskal-Wallis test followed by Dunn's multiplecomparison test). 


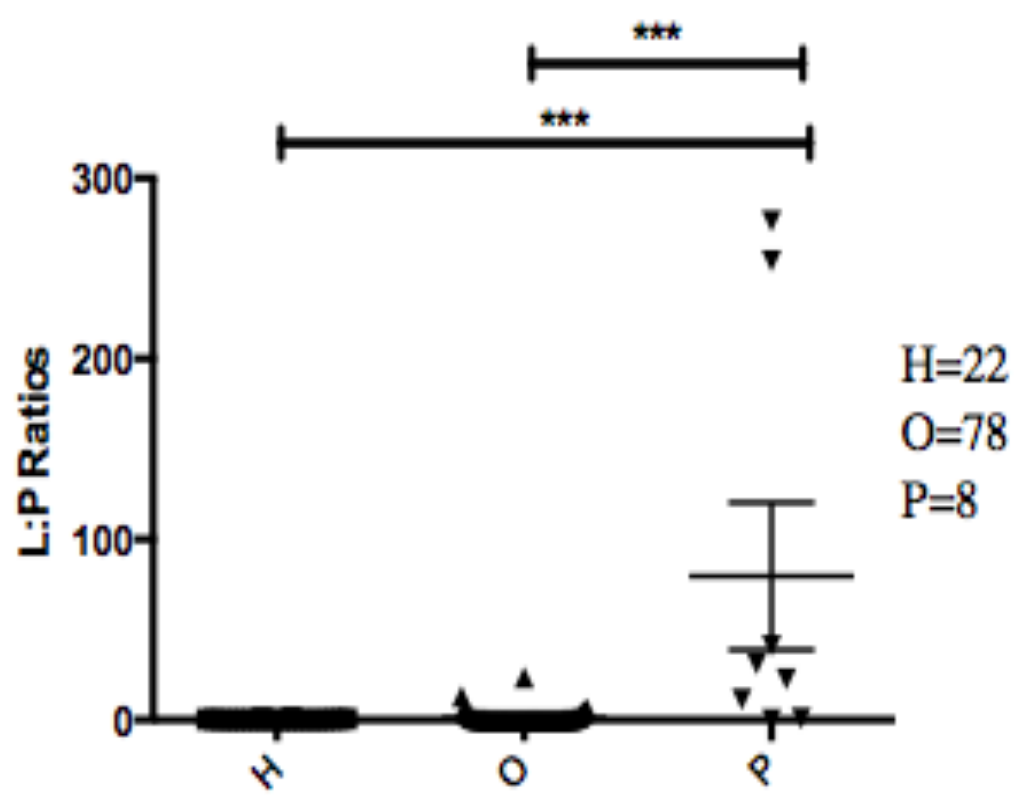


Figure 4 (on next page)

Correlation between Leptotrichiaabundance from 16S rRNA sequences and from realtime qPCR.

Cross validation of total Leptotrichia OTU abundance using real-time qPCR. After using 165 rRNA as a reference gene for normalization of the levels of Leptotrichia genus, data was normalized by fold change to three healthy controls with relatively low Leptotrichia OTU abundance. Each symbol represents a patient: $\mathrm{P}=6$, and $\mathrm{O}=12$. Leptotrichia OTU abundance was correlated with qPCR fold change according to Pearson's correlation ( $r=0.903)$. 


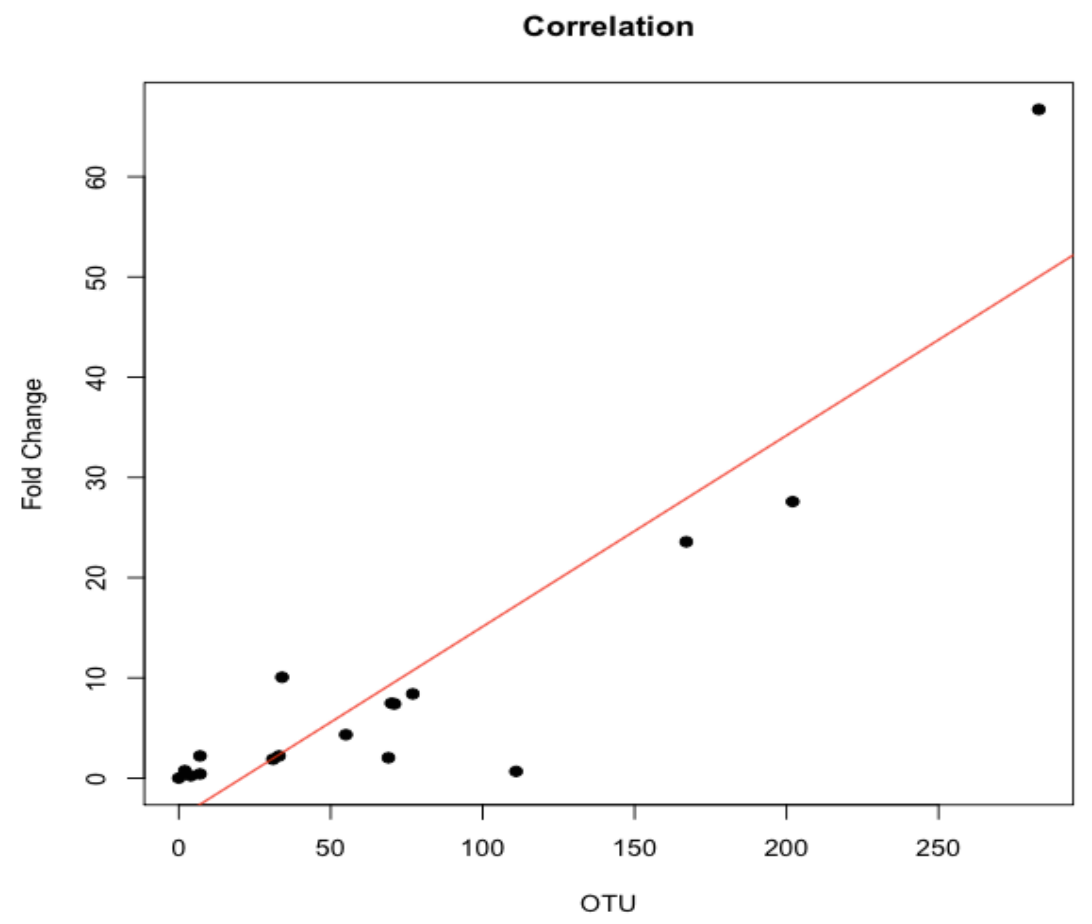


Table $\mathbf{1}$ (on next page)

Clinical characteristics of study sample $(n=108)$ 


\begin{tabular}{lcccc}
\hline Demographics & $\begin{array}{c}\text { Pancreatic Cancer (P) } \\
\mathrm{n}=8\end{array}$ & $\begin{array}{c}\text { Other Disease (O) } \\
\mathrm{n}=78\end{array}$ & $\begin{array}{c}\text { Healthy Control }(\mathrm{H}) \\
\mathrm{n}=22\end{array}$ & $\begin{array}{c}\text { Total } \\
\mathrm{n}=108\end{array}$ \\
\hline Sex & & & & \\
$\quad$ Male & 6 & 38 & 12 & 56 \\
$\quad$ Female & 2 & 40 & 10 & 52 \\
Ethnicity & & & & 77 \\
Caucasian & 6 & 56 & 15 & 13 \\
Hispanic & 2 & 6 & 5 & 5 \\
Asian & 0 & 4 & 1 & 13 \\
Unknown & 0 & 12 & 1 & \\
\hline
\end{tabular}

1 
Table 2 (on next page)

Types of identified cancers $(n=23)$ 


\begin{tabular}{lll}
\hline Cancer by Category & Age Mean & N \\
\hline Pancreas (n=8) & 71.1 & 8 \\
Pancreatic Cancer & & \\
& & \\
Digestive (n=9) & 64.7 & 3 \\
Ampullary & & 3 \\
Esophageal & & 1 \\
Stomach & & 2 \\
Rectal & & 1 \\
& 54.8 & 1 \\
Non-Digestive (n=6) & & 3 \\
Breast & & \\
Skin & & \\
Testicular & & 1 \\
Thyroid & & \\
\hline
\end{tabular}

1 